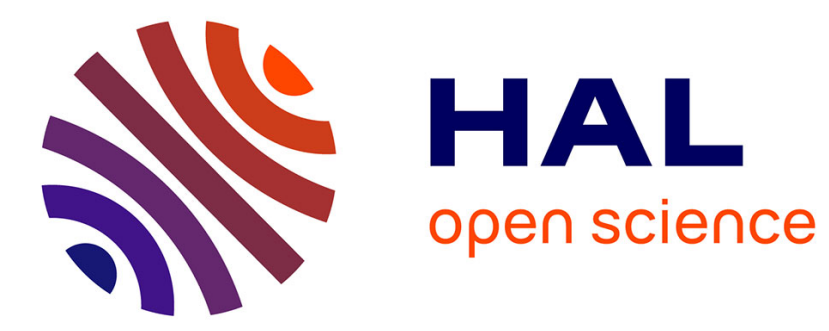

\title{
TB-LMTO method for embedded cluster
}

Vaclav Drchal, Josef Kudrnovsky

\section{- To cite this version:}

Vaclav Drchal, Josef Kudrnovsky. TB-LMTO method for embedded cluster. Philosophical Magazine, 2008, 88 (18-20), pp.2777-2786. 10.1080/14786430802238378 . hal-00513913

\section{HAL Id: hal-00513913 https://hal.science/hal-00513913}

Submitted on 1 Sep 2010

HAL is a multi-disciplinary open access archive for the deposit and dissemination of scientific research documents, whether they are published or not. The documents may come from teaching and research institutions in France or abroad, or from public or private research centers.
L'archive ouverte pluridisciplinaire $\mathbf{H A L}$, est destinée au dépôt et à la diffusion de documents scientifiques de niveau recherche, publiés ou non, émanant des établissements d'enseignement et de recherche français ou étrangers, des laboratoires publics ou privés. 


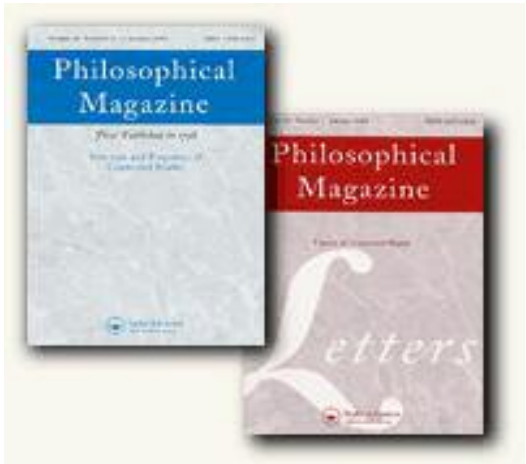

TB-LMTO method for embedded cluster

\begin{tabular}{|r|l|}
\hline Journal: & Philosophical Magazine \& Philosophical Magazine Letters \\
\hline Manuscript ID: & TPHM-08-Apr-0122 \\
\hline $\begin{array}{r}\text { Dournal Selection: } \\
\text { Author: }\end{array}$ & Philosophical Magazine \\
\hline Complete List of Authors: & $\begin{array}{l}\text { Drchal, Vaclav; Institute of Physics, Academy of Sciences } \\
\text { Kudrnovsky, Josef; Institute of Physics, Academy of Science, } \\
\text { Theory of Condensed Matter }\end{array}$ \\
\hline Keywords: & ab initio, alloys \\
\hline Keywords (user supplied): & linear muffin-tin orbital method, embedded cluster \\
\hline \\
\hline $\begin{array}{l}\text { Note: The following files were submitted by the author for peer review, but cannot be converted } \\
\text { to PDF. You must view these files (e.g. movies) online. }\end{array}$ \\
\hline embcm.tex \\
\hline
\end{tabular}

\section{ScholarONE" Manuscript Central}


Philosophical Magazine

Vol. 00, No. 00, 00 Month 200x, 1-9

\title{
RESEARCH ARTICLE
}

\section{TB-LMTO method for embedded cluster}

\author{
V. Drchal and J. Kudrnovský \\ Institute of Physics, AS CR, Na Slovance 2, CZ-182 21 Praha 8, Czech Republic \\ (Received 00 Month 200x; final version received 00 Month 200x)
}

\begin{abstract}
The method aimed at calculation of the electronic structure of an atomic cluster embedded either in an ideal crystal or in an effective medium representing a random alloy is developed on the basis of the TB-LMTO scheme. We discuss the construction of embedding potentials, the correct treatment of electrostatic interactions of the cluster with the surrounding medium, and the calculation of cluster energies. We derive the corrections to the band energy of a cluster. The method makes possible to determine the electronic structure (charge distribution, local densities of states) and magnetic properties of the cluster and to extract impurity formation energies, effective interactions between atoms and between magnetic moments. As an illustration, the method is applied to studies of magnetism of non-magnetic impurities in oxides, to calculations of the formation energies for impurities in a metal host and to determination of parameters of Bruno, Zingales and Wang model of charge transfer and screening in metallic alloys.
\end{abstract}

Keywords: linear muffin-tin orbital method; embedded cluster; random alloy; impurity formation energy; interatomic interactions; exchange interactions; charge transfer

\section{Introduction}

The embedded cluster method is a versatile tool that allows to study the electronic structure of impurities and atomic complexes embedded in a reference medium.

The idea of embedded cluster was originally used to extend the theory of disordered alloys beyond the single-site approximation [1], [2]. It became soon clear that it is suitable for studies of impurities in bulk and atoms adsorbed on solid surfaces. It was first formulated within the tight-binding approximation and later on within the Kohn, Korringa, and Rostoker method (KKR) [3], including also its fully relativistic version [4]. An important development was made by Inglesfield [5] who introduced the embedding potential for continuous spatial coordinates. The idea of embedding potential was later applied by Baraff and Schlüter to the LCAO method [6]. Among many applications of the embedded cluster method we should mention the calculation of effective interatomic interactions that determine the phase stability of alloys proposed by Gonis et al. [7]. Besides the correct embedding of electronic states of the cluster it is also important to correctly treat the electrostatic interactions with the reference system [8]. Recently the embedded cluster method was applied in studies of transport phenomena in nanostructures $[9-11]$.

The cluster calculations can be done without charge selfconsistency, i.e., using the Hamiltonian with fixed parameters (for example $[1,2,5,7]$ or a charge selfconsistent calculation for the cluster atoms can be done (for example [12-16]).

e-mail: drchal@fzu.cz, kudrnov@fzu.cz 
Here we describe the implementation of the embedded cluster approach within the tight-binding linearized-muffin-tin orbital (TB-LMTO) method and using the charge selfconsistency. The paper is organized as follows. We first develop the theoretical framework (Sec.2). We start with basic relations of the TB-LMTO method (Sec.2.1) and then we formulate, in the language of Green functions, the embedding of a cluster into the reference medium (Sec.2.2) and construct the Madelung energy and potential (Sec.2.3). Then we discuss determination of the total energy and corrections that take into account the long-range perturbation caused by the cluster (Sec.2.4). The theory is illustrated on several examples (Sec.3) which include the magnetism of non-magnetic impurities in oxides (Sec.3.1), formation energy of an impurity in a metallic host (Sec.3.2), and the determination of parameters of Bruno, Zingales and Wang model of charge transfer and screening in alloys (Sec.3.4).

\section{Theory}

\subsection{TB-LMTO method}

We first remind the basic relations of the TB-LMTO method (cf. Ref. [17]). The Hamiltonian reads as

$$
H=C+\sqrt{\Delta} S \sqrt{\Delta}
$$

where $C$ is a site-diagonal matrix of potential parameters that give the position of energy levels and $\Delta$ is is a site-diagonal matrix of potential parameters that determine the bandwidth. The matrix of screened structure constants (in representation $\alpha$ ) is denoted as $S$. The resolvent (i.e. the Green function) is given by

$$
G(z)=(z-H)^{-1}=\lambda(z)+\mu(z) g(z) \mu(z),
$$

where

$$
g(z)=(P(z)-S)^{-1}
$$

is the auxiliary Green function,

$$
P(z)=\frac{z-C}{\Delta+(\gamma-\alpha)(z-C)}
$$

is the potential function and

$$
\lambda(z)=\frac{\gamma-\alpha}{\Delta+(\gamma-\alpha)(z-C)}, \quad \mu(z)=\frac{\sqrt{\Delta}}{\Delta+(\gamma-\alpha)(z-C)}
$$

are auxiliary functions. Note that the potential function has poles for

$$
z_{p}=C-\frac{\Delta}{\gamma-\alpha}
$$

\subsection{Embedding a cluster in a reference medium}

For simplicity we will consider a cluster embedded in an infinite homogeneous crystal as a reference system (superscript 0). In order to make charge selfconsistent 
calculations feasible, we have to assume that the one-electron potentials (and, of course, the potential parameters) need to be calculated selfconsistently only for atoms in the cluster and in a certain transition region surrounding the cluster. We will assume that the potentials and potential parameters in the rest of the system are identical to those of the homogeneous reference system. Even under this approximation, the presence of a cluster will influence all lattice sites, in particular, the local densities of states, which, in turn, will influence the total number of electrons and the band term contribution to the total energy.

We divide the whole system into a cluster (denoted by the subscript $C$ ), a transition region $(T)$, and a homogeneous environment $(H)$. The cluster and the transition region form the inhomogeneous region $(I=C \cup T)$. The corresponding projection operators are denoted as $\Pi_{C}, \Pi_{T}$, and $\Pi_{H}$. It holds $\Pi_{I}=\Pi_{C}+\Pi_{T}$.

The auxiliary GF of the reference system and its projection onto the inhomogeneous region read as

$$
g^{0}=\left(P^{0}-S\right)^{-1}, \quad g_{I I}^{0}=\frac{\Pi_{I}}{P_{I I}^{0}-S_{I I}-S_{I H} \frac{\Pi_{H}}{P^{0}-S} S_{H I}}
$$

while the auxiliary GF of the system with a cluster and its projection onto the inhomogeneous region are given by

$$
g=(P-S)^{-1}, \quad g_{I I}=\frac{\Pi_{I}}{P_{I I}-S_{I I}-S_{I H} \frac{\Pi_{H}}{P-S} S_{H I}} .
$$

Because $P_{H H}=P_{H H}^{0}$, we immediately find the embedding potential

$$
\Gamma_{I I}=S_{I I}-S_{I H} \frac{H}{P^{0}-S} S_{H I}=\frac{\Pi_{I}}{P_{I I}^{0}-\frac{\Pi_{I}}{g_{I I}^{0}}}
$$

which enters the expression for the projection of the auxiliary GF onto the inhomogeneous region (8)

$$
g_{I I}=\frac{\Pi_{I}}{P_{I I}-\Gamma_{I I}}
$$

\subsection{Electrostatic energy and potential}

The Madelung potential and Madelung energy of the reference system read

$$
v_{i}^{0}=\sum_{j} M_{i j} q_{j}^{0}, \quad E^{\mathrm{Mad}, 0}=\frac{1}{2} \sum_{i j} M_{i j} q_{i}^{0} q_{j}^{0}=\frac{1}{2} \sum_{i} v_{i}^{0} q_{i}^{0}
$$

where $q_{i}^{0}$ is the net charge on the atom $i$ and the Madelung matrix is defined as

$$
M_{i j}=\left\{\begin{array}{ll}
e^{2} / R_{i j} & \text { for } i \neq j \\
0 & \text { for } i=j
\end{array} .\right.
$$

The net charges in the system with embedded cluster differ from those of the reference system by the amount

$$
\varepsilon_{i}=q_{i}-q_{i}^{0}
$$


Of course, $\varepsilon_{i} \neq 0$ only for $i \in I$. The Madelung potentials in the system with an embedded cluster are

$$
v_{i}=v_{i}^{0}+\sum_{j \in I} M_{i j} \varepsilon_{j}=v_{i}^{0}+\varphi_{i}, \quad \varphi_{i}=\sum_{j \in I} M_{i j} \varepsilon_{j}
$$

and the Madelung energy is

$$
E^{\mathrm{Mad}}=\frac{1}{2} \sum_{i j} M_{i j} q_{i} q_{j}=E^{\mathrm{Mad}, 0}+\frac{1}{2} \sum_{i \in I} \varepsilon_{i}\left(2 v_{i}^{0}+\varphi_{i}\right)
$$

\subsection{Total energy of a cluster}

The total energy of a system with embedded cluster has the standard form

$$
E^{\mathrm{tot}}=E^{\mathrm{band}}+E^{\mathrm{core}}+E^{\mathrm{dc}}+E^{\mathrm{xc}}+E^{\mathrm{Mad}} .
$$

The total energy (16) is infinite. We therefore always calculate the difference of the total energy of the system with embedded cluster and the total energy of the reference system.

The terms $E^{\text {core }}, E^{\mathrm{dc}}$, and $E^{\mathrm{xc}}$ are the sums of core energies, double-counting corrections, and exchange-correlation terms, respectively. These three contributions have single-site character and their values for sites in the homogeneous region do not depend on the cluster. Consequently, it is sufficient to calculate them only for the inhomogeneous region.

The Madelung energy $E^{\mathrm{Mad}}$ was discussed in the previous section and from the Eq. (15) it is clear that also this term can be evaluated only for the inhomogeneous region.

The band term $E^{\text {band }}$

$$
E^{\text {band }}=\int^{E_{F}} d E E n(E)=\frac{1}{2 \pi i} \int_{C_{R}} d z z \operatorname{Tr} G(z)
$$

is more difficult to evaluate because it depends on the density of states $n(E)$

$$
n(E)=-\frac{1}{\pi} \operatorname{Im} \operatorname{Tr} G(E+i 0), \quad G(z)=(z-H)^{-1}
$$

which contains Tr over the whole space. The presence of the cluster leads to changes of the Green function $G(z)$ not only in the inhomogeneous region, but also in the homogeneous region. The changes of $G(z)$ in the homogeneous region can be included using the partitioning technique applied to the $\operatorname{Tr} \log G(z)$ in the following way. The band term can be written in the form

$$
E^{\text {band }}=E_{F} N_{e l}+\frac{1}{2 \pi i} \int_{C_{R}} d z \operatorname{Tr} \log (z-H)
$$

where the integration contour is defined as

$$
C_{R}=\left\{z: z=E_{F}-R+R e^{i \phi}, \phi \in(0,2 \pi)\right\} .
$$


The partitioning gives

$$
\begin{aligned}
\operatorname{Tr} \log (z-H)= & \operatorname{Tr}_{I} \log \left(z \Pi_{I}-H_{I I}-H_{I H} \frac{\Pi_{H}}{z-H} H_{H I}\right) \\
& +\operatorname{Tr}_{H} \log \left(z-H_{H H}\right) .
\end{aligned}
$$

The terms $\operatorname{Tr}_{H} \log \left(z-H_{H H}\right)$ are identical for the reference system and for the cluster and thus will mutually subtract when we calculate the difference of total energies. The application of this elegant trick is not straightforward, because (i) logarithm is a multivalued function and, (ii) the potential function $P(z)$ in the TB-LMTO has poles $z_{p}$, see Eq.(6). The first obstacle may be overcome by careful monitoring phases of the log function, but the second obstacle is a serious one if the poles $z_{p}$ lie inside or close to the integration path. In such a case one cannot use the trick with $\operatorname{Tr} \log G(z)$. Instead, it is necessary to calculate

$$
E_{I}^{\text {band }}=\int^{E_{F}} d E E n_{I}(E), \quad n_{I}(E)=-\frac{1}{\pi} \operatorname{Im} \operatorname{Tr}_{I} G(E+i 0)
$$

and to employ sufficently large inhomogeneous region in order to include the effects of the perturbation by the cluster with the required accuracy.

\subsection{Remarks}

At this stage a few comments are in place.

(i) So far we have discussed the simplest case in which the reference system was an ideal crystal. Along similar lines one can also assume a random alloy as a reference medium. The main difference is that it is necessary to solve a set of mutually coupled CPA equations for atoms in the transition region. In addition, to make the problem tractable, the vertex corrections corresponding to coupled scattering on the cluster and on the disorder in the homogeneous region have to be neglected. The method is also suitable for treatment of isolated clusters, it is sufficient to use empty spheres as a reference system. Finally, the clusters on the surface of a solid can be studied if the reference medium consists of an ideal semi-infinite solid, either a crystal or an alloy.

(ii) The efficiency of the method requires that the inhomogeneous region is as small as possible. It is therefore necessary to have short-ranged structure constants. This leads naturally to the choice of the TB-LMTO method. It is necessary to check the convergence of results with the size of the cluster.

(iii) The calculation proceeds in two steps: First, the embedding potential $\Gamma_{I I}$ and the Madelung potential $v_{i}^{0}$ of the reference medium for atoms in the inhomogeneous region are calculated and then the selfconsistent calculation is performed for the inhomogeneous region.

\section{Applications}

\subsection{Magnetism of non-magnetic impurities in non-magnetic oxides}

Somewhat surprisingly, magnetism can be observed in materials without magnetic atoms. It is referred to as " $\mathrm{d}^{0}$, or intrinsic ferromagnetism. The experiments have shown that for example thin films of $\mathrm{HfO}_{2}$ and $\mathrm{ZrO}_{2}$ can have Curie temperature well above the room temperature [18], [19]. Magnetism was also reported in 
irradiated graphite [20], [21] and in La-doped hexaborides such as $\mathrm{CaB}_{6}$ [22]. Ferromagnetism was predicted theoretically in materials with flat p-bands crossing the Fermi energy [23].

Here we will discuss the non-magnetic transition metal oxides such as $\mathrm{TiO}_{2}$, $\mathrm{ZrO}_{2}, \mathrm{HfO}_{2}$. The original hypothesis that oxygen vacancies are responsible for magnetism in these materials was not confirmed by the DFT calculations [24]. On the other hand, the cation vacancies can induce magnetic moments on neighboring oxygen atoms. The formation energy of this type of defects is rather high so their concentration will be very low under equilibrium conditions, which means that they cannot cause magnetism. The $\mathrm{Ti}, \mathrm{Zr}$, and $\mathrm{Hf}$ are four-valent atoms in dioxides such as $\mathrm{ZrO}_{2}$. The substitution by an alkali or alkali earth metal could lower the formation energy and still polarize neighboring oxygen atoms.

We tested this hypothesis for $\mathrm{ZrO}_{2}$ host and made calculations for clusters (see Table 1) that contained 59 sites with the central site occupied either by a vacancy (Vac) or by a potassium atom $(\mathrm{K})$. We have assumed the fluorite structure for $\mathrm{ZrO}_{2}$. The results are given in Table 2.

Table 1. The cluster in $\mathrm{ZrO}_{2}$ containing 59 atoms used in present calculations. The distance $R$ from the origin is expressed as a multiple of $\mathrm{CaF}_{2}$ lattice constant $a$ and $n$ denotes the number of atoms in a given coordination shell.

\begin{tabular}{ccrl}
\hline shell & $R$ & $n$ & atom \\
\hline 0 & $0.0000 a$ & 1 & Zr, Vac, K, etc. \\
1 & $0.4330 a$ & 8 & O ... O (1) \\
2 & $0.5000 a$ & 6 & empty \\
3 & $0.7071 a$ & 12 & Zr \\
4 & $0.8292 a$ & 24 & O ... O $(2)$ \\
5 & $0.8660 a$ & 8 & $\mathrm{E}$ \\
\hline
\end{tabular}

Table 2. Calculated magnetic moments $m$ in units $\mu_{B}$ in clusters with a vacancy and with a $\mathrm{K}$ atom at the central site. For comparison we give also moments found in FPLAPW calculations for supercells $\mathrm{VacZ}_{7} \mathrm{O}_{16}(12.5$ \%), $\mathrm{VacZr}_{31} \mathrm{O}_{64}(3.125 \%), \mathrm{KZr}_{7} \mathrm{O}_{16}(12.5 \%)$, and $\mathrm{KZr}_{31} \mathrm{O}_{64}$ (3.125\%) (see Ref. [25]).

\begin{tabular}{cccc|cccc}
\hline & cluster & $12.5 \%$ & $3.125 \%$ & & cluster & $12.5 \%$ & $3.125 \%$ \\
& $m\left[\mu_{B}\right]$ & $m\left[\mu_{B}\right]$ & $m\left[\mu_{B}\right]$ & & $m\left[\mu_{B}\right]$ & $m\left[\mu_{B}\right]$ & $m\left[\mu_{B}\right]$ \\
\hline $\mathrm{Vac}$ & 0.005 & 0.00 & 0.00 & $\mathrm{~K}$ & 0.158 & 0.10 & 0.08 \\
$\mathrm{O}(1)$ & 0.421 & 0.40 & 0.38 & $\mathrm{O}(1)$ & 0.244 & 0.30 & 0.21 \\
$\mathrm{O}(2)$ & 0.027 & 0.05 & 0.03 & $\mathrm{O}(2)$ & 0.023 & 0.05 & 0.03 \\
total & 3.927 & 3.81 & 4.00 & total & 2.616 & 2.99 & 2.67 \\
\hline
\end{tabular}

\subsection{Impurity formation energy}

As an example consider a Zn impurity in fcc copper. Its formation energy is defined as

$$
\Delta E=\left(E\left[\mathrm{Cu}_{N-1} \mathrm{Zn}\right]+\epsilon[\mathrm{Cu}]\right)-\left(E\left[\mathrm{Cu}_{N}\right]+\epsilon[\mathrm{Zn}]\right)
$$

where $E\left[\mathrm{Cu}_{N-1} \mathrm{Zn}\right]$ is the energy of a cluster formed of $N-1 \mathrm{Cu}$ atoms and a $\mathrm{Zn}$ impurity while $E\left[\mathrm{Cu}_{N}\right]$ is the energy of a cluster of $N \mathrm{Cu}$ atoms. The quantities $\epsilon[\mathrm{Cu}]$ and $\epsilon[\mathrm{Zn}]$ denote the energies of isolated $\mathrm{Cu}$ and $\mathrm{Zn}$ atoms, respectively. This example allows us to demonstrate the efficiency of the trick with the Tr log. In figure 1 we compare the formation energy of a $\mathrm{Zn}$ impurity in fcc copper calculated 
directly and by using the $\operatorname{Tr} \log$. It is clearly seen that when using the $\operatorname{Tr} \log$ it is sufficient to include the impurity site and its nearest neighbors while in direct calculation one has to include several coordination shells in order to achieve a comparable accuracy.

Figure 1. Formation energy of a $\mathrm{Zn}$ impurity in fcc $\mathrm{Cu}$ as a function of cluster size.

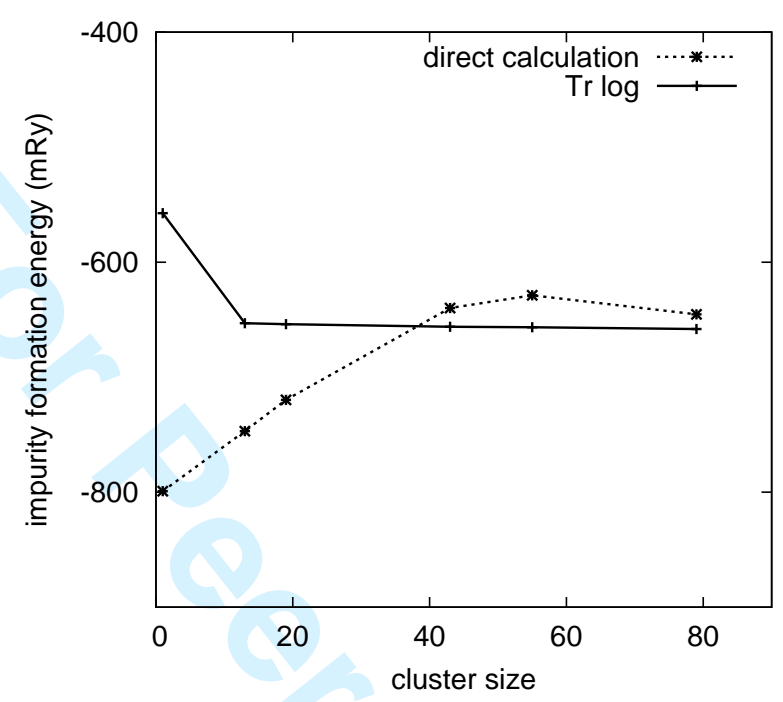

\subsection{Induced charges}

The charges induced in several coordination spheres around the Zn impurity in fcc-Cu are shown in figure 2. The induced charges decrease quite fast with distance from the central atom particularly beyond the second coordination shell due to the efficient screening in the metallic system.

Figure 2. Induced charges (per coordination shell) around the $\mathrm{Zn}$ impurity in fcc $\mathrm{Cu}$.

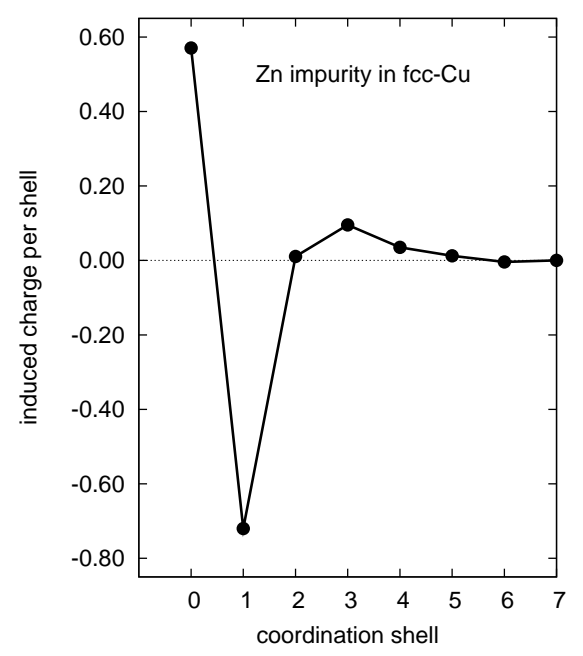




\subsection{Parameters of $B Z W$ model}

Bruno, Zingales, and Wang (BZW) proposed a model for description of charge transfer and screening in alloys [26]. The Hamiltonian of the BZW model reads

$$
E\left(\left\{q_{i}\right\}\right)=E^{\mathrm{loc}}\left(\left\{q_{i}\right\}\right)+E^{\mathrm{Mad}}\left(\left\{q_{i}\right\}\right)=\sum_{i} \frac{a_{i}}{2}\left(q_{i}-b_{i}\right)^{2}+\frac{1}{2} \sum_{i, j} M_{i j} q_{i} q_{j}
$$

where $q_{i}$ is the net charge at the site $i, b_{i}$ is the bare charge, and $a_{i}$ is the strength of the electron-electron interaction. Minimization of energy gives

$$
a_{i}\left(q_{i}-b_{i}\right)+\sum_{j} M_{i j} q_{j}=0, \quad V_{i}=\sum_{j} M_{i j} q_{j}
$$

which is equivalent to the well-known linear relation [27] between the net charge and local Madelung potential $V_{i}$ (the so-caled q-V relation)

$$
a_{i} q_{i}+V_{i}=a_{i} b_{i}=k_{i}
$$

The BZW model can be used to determine the screened Madelung interactions $M_{i j}^{\mathrm{scr}}$, the electrostatic corrections to effective interatomic interactions

$$
V_{i j}^{\mathrm{scr}}=M_{i j}^{\mathrm{scr}}\left(q_{i}^{A}-q_{i}^{B}\right)\left(q_{i}^{A}-q_{i}^{B}\right)
$$

that serve as parameters of the effective Ising Hamiltonian of the alloy, and to find the statistics of net charges on atoms in an alloy [28].

The parameters $a_{i}, b_{i}$ of the BZW model can be determined from the supercell calculations [26], from the local-field CPA [29], and also from the calculations for embedded clusters. To find the parameters $a_{i}, b_{i}$ we add an external Madelung potential $V_{0}^{\text {ext }}$ on the site at origin and calculate the charge $q_{0}$ and the total potential $V_{0}^{\text {tot }}=V_{0}^{\text {ext }}+V_{0}^{\text {induced }}$ at the site at origin. These calculations are done for several values of the external potential $V_{0}^{\text {ext }}$. The charges $q_{0}$ and potentials $V_{0}^{\text {tot }}$ fulfill the $\mathrm{q}-\mathrm{V}$ relation

$$
a q_{0}+V_{0}^{\text {tot }}=k=a b
$$

from which one can extract the parameters $a_{i}$ and $b_{i}$. The values obtained for bcc$\mathrm{Cu}_{50} \mathrm{Zn}_{50}$ alloy are given in Table 3 and compared with values obtained from KKR calculation for large supercell in Ref. [30]. The difference in values obtained by two different methods can be explained by a different size of muffin spheres used in the LMTO and in the KKR.

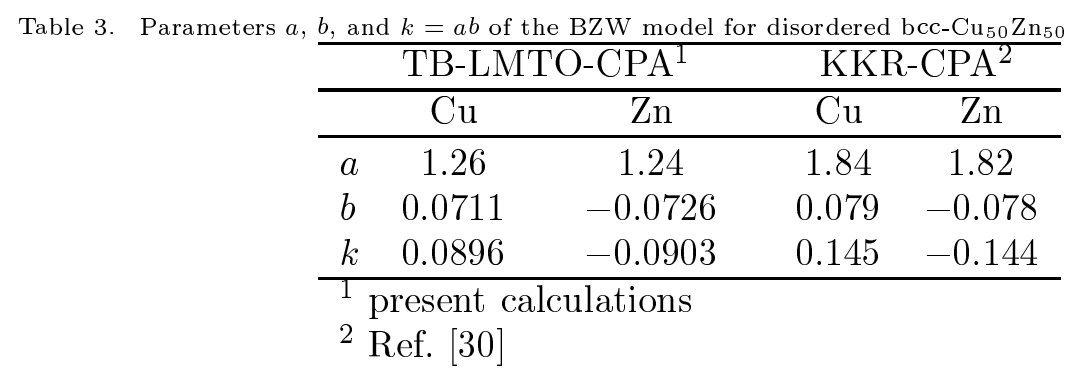




\section{Conclusions and outlook}

To summarize, we have developed the embedded cluster method within the framework of the tight-binding muffin-tin orbital method (TB-LMTO). It turns out that it is a flexible, numerically medium-cost method that can yield the electronic and magnetic structure of impurities, finite clusters, complexes, etc., i.e., the charge distributions, local densities of states, and magnetic moments. It also allows to study the energetics of solids and imperfections, namely, the quantities such as impurity or complex formation energies, effective interatomic interactions (parameters of Ising type Hamiltonian), effective exchange interactions (parameters of Heisenberg Hamiltonian), and the parameters of Bruno, Zingales, and Wang model of screening and charge transfer.

\section{Acknowledgments}

The research was carried out within the project AVOZ10100520 of the Academy of Sciences of the Czech Republic. Financial support was provided by the Grant Agency of the Academy of Sciences of the Czech Republic (Project A100100616).

\section{References}

[1] L. Schwartz and H. Ehrenreich: Phys. Rev. B 6 (1972), 2923.

[2] A. Gonis and J. W. Garland: Phys. Rev. B 16 (1977), 2424.

[3] A. Gonis, G. M. Stocks, W. H. Butler, and H. Winter: Phys. Rev. B 29 (1984), 555.

[4] A. Gonis, P. Weinberger: Phys. Rev. B 37 (1988), 1383.

[5] J. E. Inglesfield: J. Phys. C: Solid State Phys. 14 (1981), 3795.

[6] G. A. Baraff and M. Schlüter: J. Phys. C: Solid State Phys. 19 (1986), 4383.

[7] A. Gonis et al., Phys. Rev. B 36 (1987), 4630.

[8] B. Lazarovits, L. Szunyogh, and P. Weinberger: Phys. Rev. B 65 (2002), 104441.

[9] K. Palotás, B. Lazarovits, L. Szunyogh, and P. Weinberger: Phys. Rev. B 67 (2003), 174404.

[10] J. E. Inglesfield, S. Crampin, and H. Ishida: Phys. Rev. B 71 (2005), 155120.

[11] D. Wortmann, H. Ishida, and S. Blügel: Phys. Rev. B 72 (2005), 235113.

[12] T. B. Grimley and G. Pisani: J. Phys. C: Solid State Phys. 7 (1974), 2831.

[13] G. Pisani: Phys. Rev. B 17 (1978), 3143.

[14] D. E. Ellis, G. A. Benesh, and E. Byrom: Phys. Rev. B 20 (1979), 1198.

[15] J. E. Inglesfield: J. Phys. F: Metal Phys. 11 (1981), L287.

[16] P. J. Braspenning, R. Zeller, A. Lodder, and P. H. Dederichs: Phys. Rev. B 29 (1984), 703.

[17] I. Turek, V. Drchal, J. Kudrnovský, M. Šob, and P. Weinberger: Electronic Structure of Disordered Alloys, Surfaces and Interfaces, Kluwer Academic, (Boston, London, Dordrecht, 1977).

[18] M. Venkatesan, C. B. Fitzgerald, and J. M. D. Coey: Nature (London) 430 (2004), 630.

[19] M. Venkatesan, C. B. Fitzgerald, J. G. Luney, and J. M. D. Coey: Phys. Rev. Lett. 93 (2004), 177206.

[20] T. L. Makarova et al. : Nature (London) 413 (2001), 716.

[21] P. Esquinazi et al.: Phys. Rev. B 66 (2002), 024429.

[22] D. P. Young et al. : Nature (London) 397 (1999), 412.

[23] M. Sieberer, J. Redinger, S. Khmelevskyi, and P. Mohn: Phys. Rev. B 73 (2006), 024404.

[24] C. Das Pemmaraju and S. Sanvito: Phys. Rev. Lett. 94 (2005), 217205.

[25] F. Máca, J. Kudrnovský, V. Drchal, and G. Bouzerar: arXiv:0711.0280v1 (2007).

[26] E. Bruno, L. Zingales, and Y. Wang: Phys. Rev. Lett. 91 (2003), 166401.

[27] J. S. Faulkner, Y. Wang, and G. M. Stocks: Phys. Rev. B 52 (1995), 17106.

[28] V. Drchal, R. Hammerling, and P. Weinberger: Phys. Rev. B 74 (2006), 214202.

[29] E. Bruno, L. Zingales, and A. Milici: Phys. Rev. B 66 (2002), 245107.

[30] J. S. Faulkner, Y. Wang, and G. M. Stocks: Phys. Rev. B 55 (1997), 7492. 


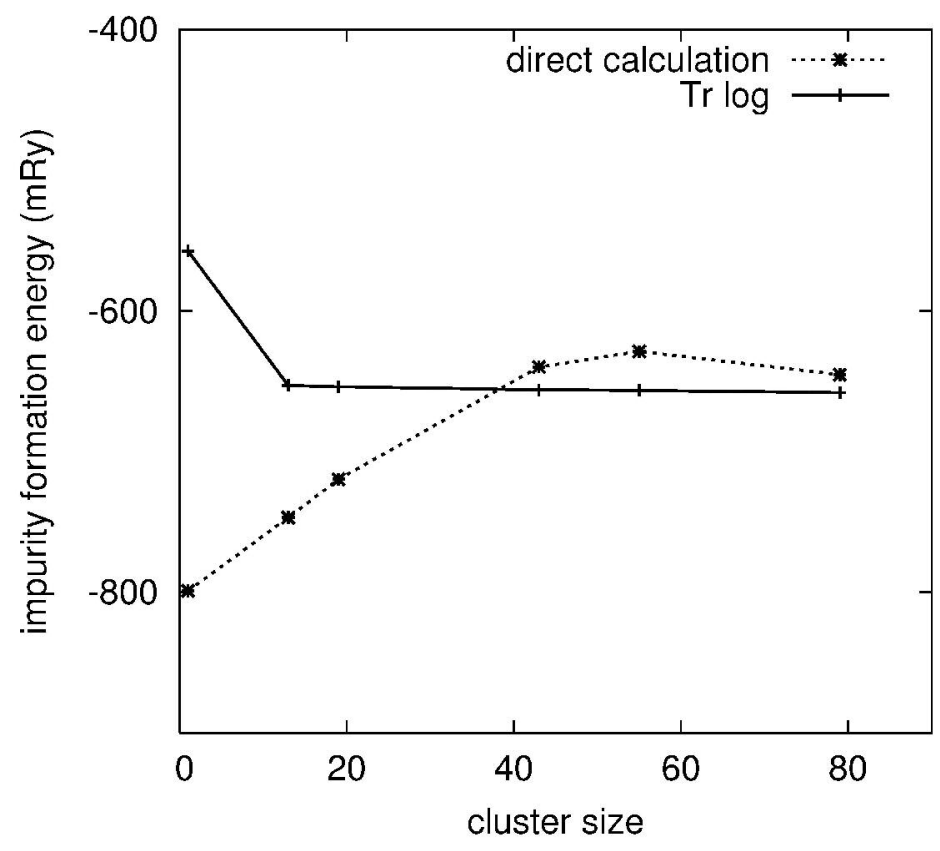

$215 \times 279 \mathrm{~mm}(600 \times 600 \mathrm{DPI})$ 


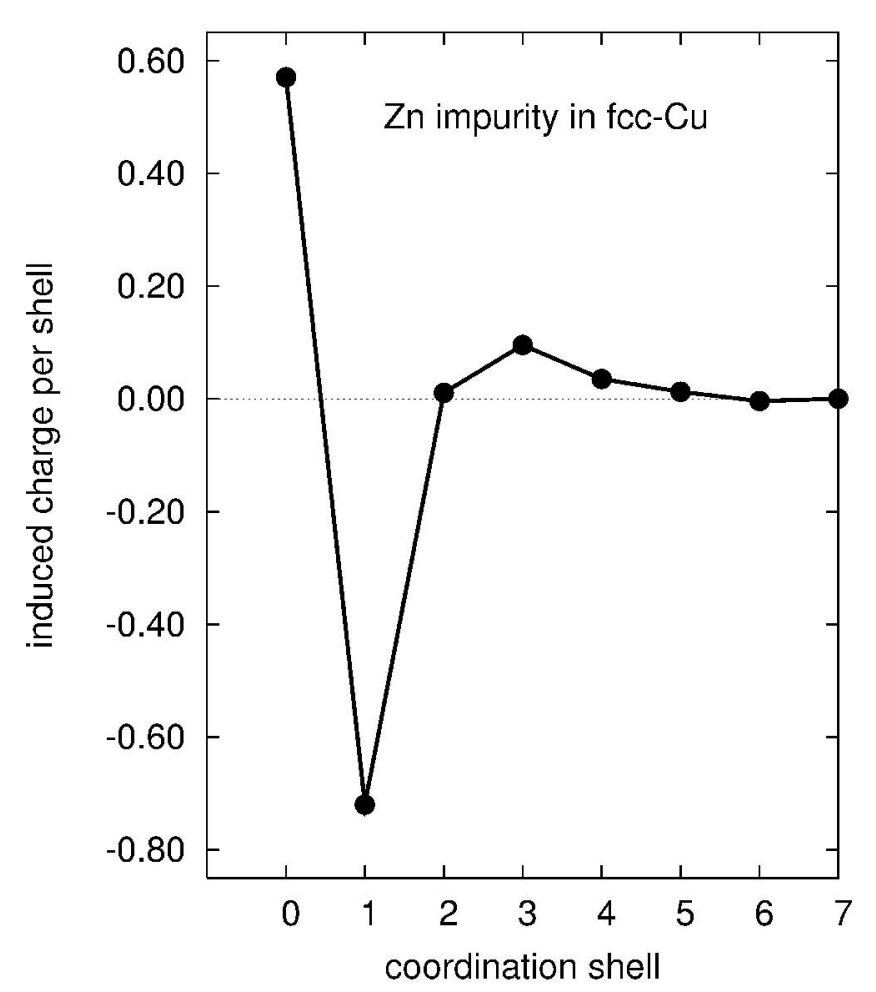

$215 \times 279 \mathrm{~mm}(600 \times 600 \mathrm{DPI})$ 